\title{
Wavelength tuning, chirping, and thermally induced decay of type IA FBGs using purely optical methods
}

K. Kalli, George Simpson, Kaiming Zhou, Lin Zhang, David Birkin, et al.

K. Kalli, George Simpson, Kaiming Zhou, Lin Zhang, David Birkin, Tim Ellingham, lan Bennion, "Wavelength tuning, chirping, and thermally induced decay of type IA FBGs using purely optical methods," Proc. SPIE 5855, 17th International Conference on Optical Fibre Sensors, (23 May 2005); doi: $10.1117 / 12.624289$

SPIE Event: 17th International Conference on Optical Fibre Sensors, 2005, Bruges, Belgium 


\title{
Wavelength tuning, chirping and thermally induced decay of type IA FBGs using purely optical methods
}

\author{
Kyriacos Kalli* a , George Simpson ${ }^{\mathrm{b}}$, Kaiming Zhou ${ }^{\mathrm{b}}$, Lin Zhang ${ }^{\mathrm{b}}$, David Birkin ${ }^{\mathrm{b}}$, Tim Ellingham ${ }^{\mathrm{b}}$, \\ Ian Bennion ${ }^{\mathrm{b}}$ \\ ${ }^{a}$ Higher Technical Institute, C. Kavafi Street, Nicosia, 2152 Cyprus \\ ${ }^{\mathrm{b}}$ Photonics Research Group, Aston University, Birmingham B4 7ET United Kingdom
}

\begin{abstract}
We report experimental measurements of reversible wavelength tuning, chirping and permanent thermally induced decay of type1A fibre Bragg gratings using purely optical means. The grating properties are modified in a controlled manner by utilising the characteristics of the intrinsic $1400 \mathrm{~nm}$ absorption band of the optical fibre and two tuneable lasers coincident with that band. It is shown that the type 1A grating can be selectively tuned in a grating network and that selective tuning can be attained in a common section of suitably prepared optical fibre. Applications of this approach for tuning fibre lasers or edge filter modification in sensing applications are discussed.
\end{abstract}

Keywords: Optical fibre sensors, fibre Bragg gratings, type 1A grating, photosensitivity, wavelength tuning

\section{INTRODUCTION}

Type IA fibre Bragg gratings may be considered a subtype of Type I gratings and are typically formed after the prolonged UV exposure of a standard grating in hydrogenated germanosilicate fibre ${ }^{1,2}$, although recent improvements in their inscription have shown that they can be readily inscribed in a suitably prepared optical fibre ${ }^{3}$. The spectral characteristics of type IA gratings are unique; they are distinct from other grating types as they exhibit a large increase in the mean core index that is identifiable as a large red shift seen in the Bragg wavelength ( inscription, Figure 1. This red shift is dependent on fibre type and hydrogenation conditions, but for a highly doped fibre is typically in the order of $15-20 \mathrm{~nm}$, and for SMF28 is 5-8nm. The maximum wavelength shift translates to an increase in the mean index of up to $2 \times 10^{-2}$. More importantly, IA gratings have been shown to exhibit the lowest temperature coefficient of all grating types reported to date, which makes them ideal for use in a temperature compensating, dual grating sensor, as has recently been demonstrated ${ }^{4}$. Figure 2 shows the spectrum of a $4 \mathrm{~mm}$ regenerated IA grating and a $1 \mathrm{~mm}$ standard Type I grating. These gratings have been written in the same fibre with the same phase mask, yet their central reflecting peaks are $14.5 \mathrm{~nm}$ apart after annealing. The large increase in mean refractive index increases the fibre NA to such an extent that it becomes few-moded; the NA of the virgin fibre is 0.161 , compared to 0.247 for the modified fibre, accounting for the transmission spectrum ghost mode.

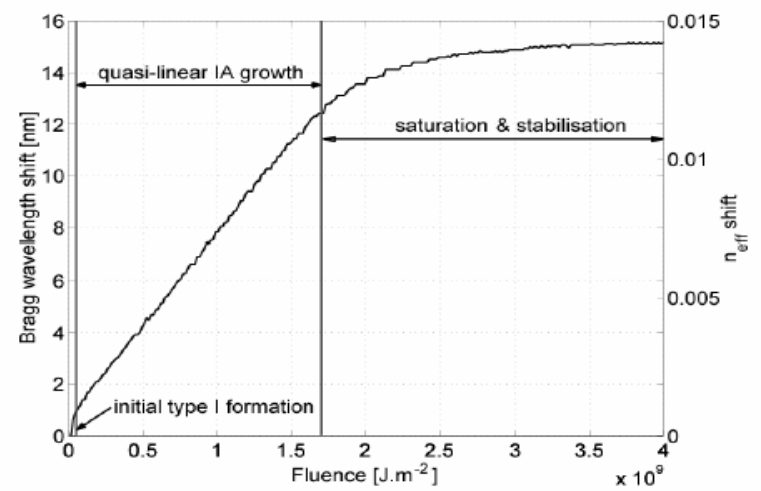

Fig. 1. Characteristic red shift during prolonged UV exposure of hydrogenated germanosilicate fibre shown in terms of the spectral red shift and associated refractive index change.

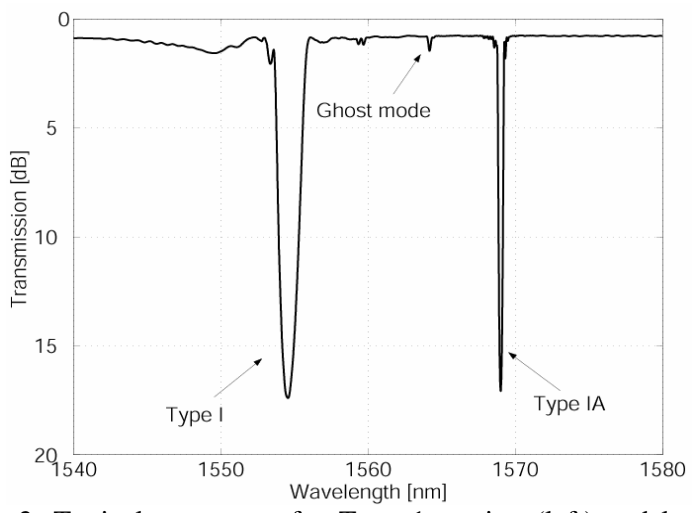

Fig. 2. Typical spectrum of a Type 1 grating (left) and longer Type IA grating (right) written in the same fibre with the same phase mask, but displaying a $14 \mathrm{~nm}$ wavelength difference.

*kkalli@hti.ac.cy; phone +35722406537; fax +35722406545; www.hti.ac.cy 


\section{ABSORPTION BAND CHARACTERISTICS AT 1400NM}

We have previously shown that there is a strong correlation between the growth of the $\mathrm{OH}$ absorption band formation in the optical fibre during prolonged UV exposure and the increase in the mean index change of the fibre grating ${ }^{3}$. Figure 3 confirms the parallel mean index evolution, akin to the maturity level of a type IA grating, and the associated increase in the absorption band at $1400 \mathrm{~nm}$. Both curves follow the three trends of Figure 1. This absorption band is a consequence of the formation of $\mathrm{OH}$ ions within the fibre and has no dependence on the modulated index change required for a Bragg grating to be formed in the core ${ }^{5}$. It is therefore possible to monitor the transmission loss at $1400 \mathrm{~nm}$ and glean accurate feedback as to the maturity of the fibre. When the absorption loss saturates, the fibre is fully mature and ready for inscription of any form of IA grating, be it slanted, long period or standard. The peak absorption band loss at $\sim 1400 \mathrm{~nm}$ is typically $\sim 1.2 \mathrm{dBmm}^{-1}$ for a $\mathrm{B} / \mathrm{Ge}$ co-doped fibre. Therefore, if the fibre is pumped with a laser source that coincides with the $1400 \mathrm{~nm}$ absorption band the fibre will be heated in the region where there is light absorption, e.g. the location of the Type IA grating.

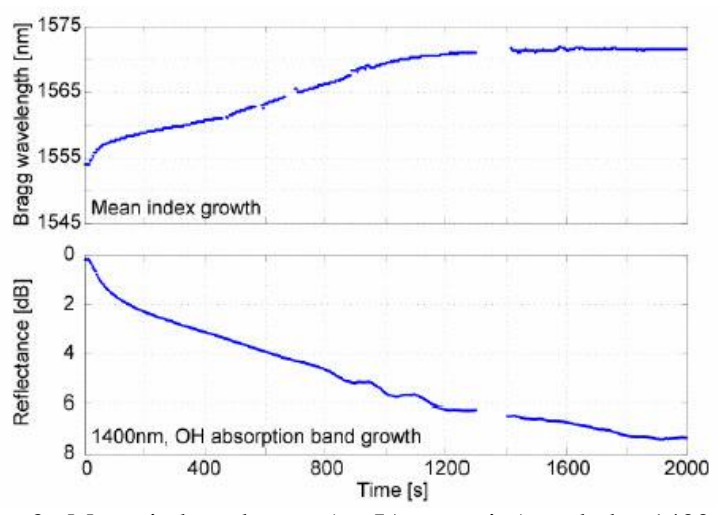

Fig. 3. Mean index change (or IA maturity) and the $1400 \mathrm{~nm}$ absorption band under UV exposure. This enables IA index changes to be inscribed without first writing a grating as the index change may be monitored by observing the $1400 \mathrm{~nm}$ band.

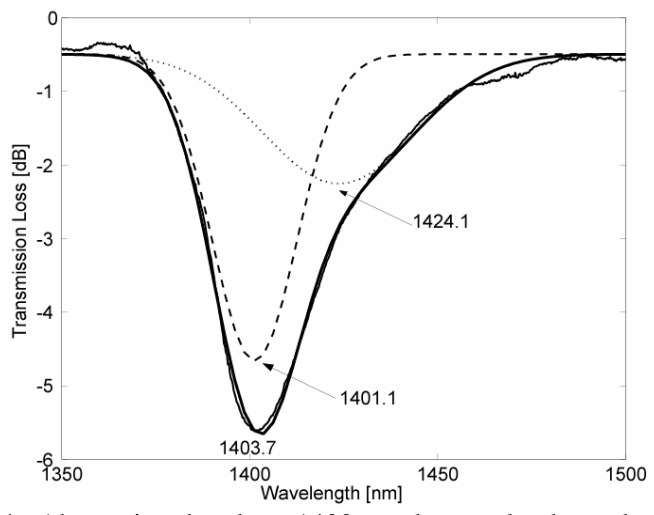

Fig. 4. Absorption band at $1400 \mathrm{~nm}$ deconvolved to show its constituent peaks. Mixture of $\mathrm{SiOH}(1390 \mathrm{~nm}) \& \mathrm{GeOH}$ $(1410 \mathrm{~nm})$ bands - biased in favour of $\mathrm{GeOH}$ under UV exposure conditions.

\section{REVERSIBLE WAVELENGTH TUNING}

In the experiment highlighted in Fig. 5 the IA-I dual grating sensor was illuminated with a broadband source in the spectral region of the gratings and monitored in reflection by the OSA. Powerful tuneable lasers were used to coincide with the $1400 \mathrm{~nm}$ absorption band. The IA-I grating consisted of a $2 \mathrm{~mm}$ IA grating, manufactured by the blank beam UV pre-exposure method directly adjacent to a $2 \mathrm{~mm}$ Type I grating ${ }^{4}$. Two lasers were used for Type $1 \mathrm{~A}$ modification; the first was a $10 \mathrm{~mW}$ Santec laser with a range of $1410 \mathrm{~nm}$ to $1490 \mathrm{~nm}$, set to coincide with the peak of the absorption band. The second laser was a $5 \mathrm{~W}$ Raman pump operating at $1425 \mathrm{~nm}$, sitting at the edge of the band and coincident with the feature at $1424 \mathrm{~nm}$.

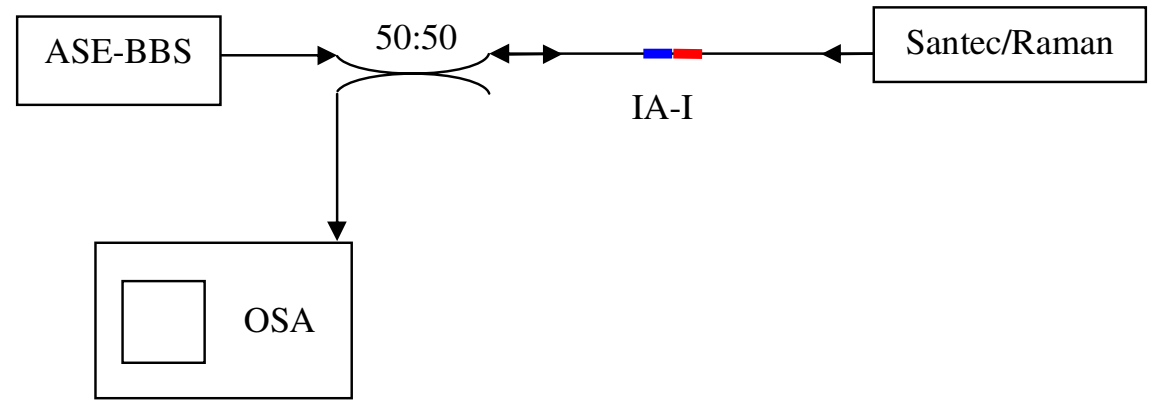

Fig. 5. Set-up for Type 1A laser based modification 


\subsection{Type $1 \mathrm{~A}$ wavelength tuning - low power, near absorption peak}

Figures 6 shows the shift in в of this grating with increasing laser power at 1410nm. Unfortunately a limiting factor is the minimum resolvable wavelength change of the OSA as shown by the pixelated data. In Figure 7 the wavelength of the tuneable laser is shifted (1410nm to $1490 \mathrm{~nm}$ ) whilst maintaining a constant output power (10mW) and the shape of the $\mathrm{OH}$ band is used as an edge filter, varying the amount of energy absorbed and hence the tuning of ${ }_{\mathrm{B}}$.

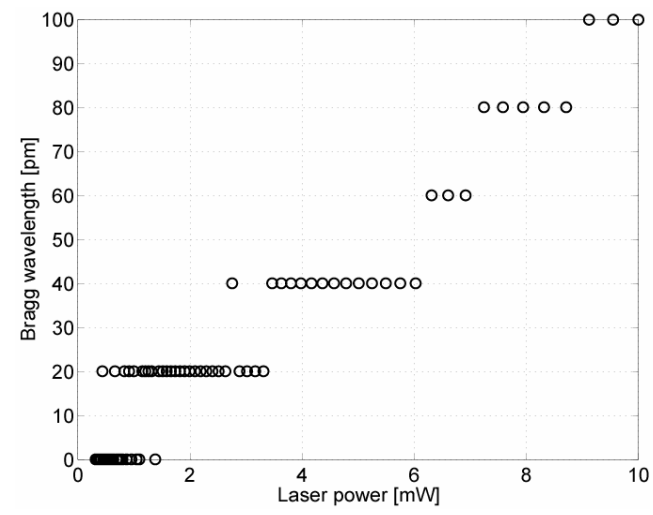

Fig. 6. Shift in the Bragg wavelength of a IA grating heated with a $1410 \mathrm{~nm}$ tuneable laser of varying power levels.

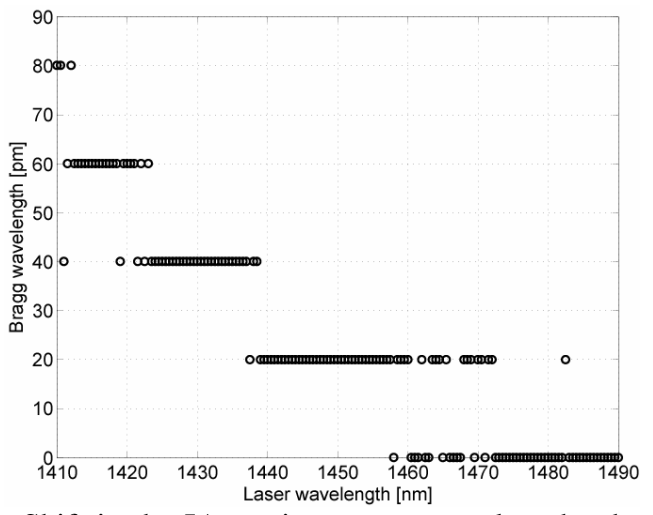

Fig. 7. Shift in the IA grating centre wavelength when the tuneable laser is held at constant power and the wavelength shifted up the edge of the $\mathrm{OH}$ band.

\subsection{Type 1A wavelength tuning - high power, far from absorption peak}

The experiment was reconfigured so that the Raman source illuminated the IA-I gratings. Although the gratings are directly adjacent to one another, the fibre portion that was pre-exposed to UV radiation and contains the higher wavelength IA grating only absorbs the $1425 \mathrm{~nm}$ radiation; the Type I grating written in virgin fibre with no strong $1400 \mathrm{~nm}$ absorption band is not heated. The negligible thermal mass of the fibre prevents any significant levels of conduction from the two regions. Thus the application of the Raman laser causes the IA grating to spectrally shift whilst the Type I grating maintains a fixed wavelength. Table 1 summarises the relative shift of gratings. Figure 8 indicates a linear relationship between Raman power and wavelength shift. Note that the slight fluctuation in the wavelength of the gratings may be attributed to variations in the ambient temperature.

\begin{tabular}{c:cc}
\hline $\begin{array}{c}\text { Raman Power } \\
\mathrm{mW}\end{array}$ & $\Delta \lambda_{B R}^{I A}$ & $\Delta \lambda_{B R}^{I}$ \\
$\mathrm{pm}$ & $\mathrm{pm}$ \\
\hline 0 & 0 & -3 \\
25.1 & 105 & 0 \\
52.9 & 245 & 17 \\
79.22 & 367 & 17 \\
103.45 & 455 & -3 \\
124.8 & 525 & 17 \\
\hline
\end{tabular}

Table 1. Wavelength shifts of the Type I and IA gratings when heated with a Raman source at $1425 \mathrm{~nm}$.

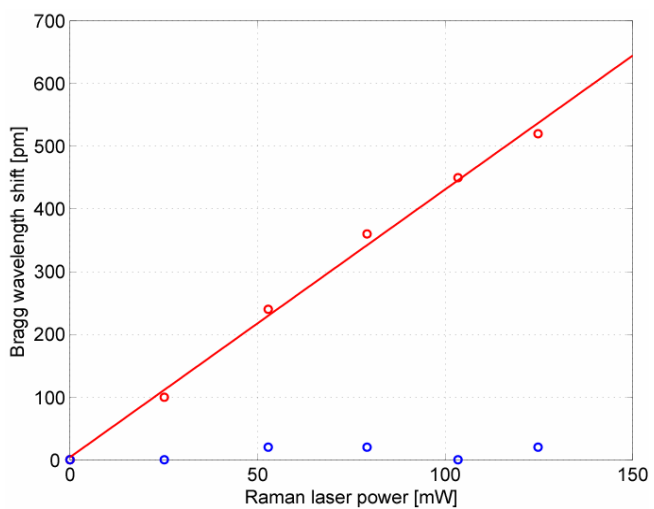

Fig. 8. Wavelength shifts of the Type I and IA gratings when pumped with a Raman source at $1425 \mathrm{~nm}$.

\section{INDUCING CHIRP AND PERMANENT DECAY}

If we next consider the effect of the Raman source being absorbed along the length of the grating we note that the heating effect will be non-uniform along the length of the absorbing section of fibre and that the result will be an induced 
chirp along the grating. Clearly higher Raman powers will induce a larger chirp and we should see an increase in the FWHM reflectance bandwidth. The Raman source intensity decay along the IA grating length is

$$
I(p)=I(p-1) \cdot\left(1-10^{\frac{A}{10}}\right) \propto \Delta T
$$

where $I(p)$ is the Raman intensity at a point $p, A$ is the absorption per unit length of the Raman radiation measured in $\mathrm{dB}$ and $T$ is the induced temperature increase. Figure 9 indicates the linearity between the FWHM bandwidth with the Raman intensity, for which we note that the central wavelength also shifts. Finally, Figure 10, we observe that under continued laser pumping at $1425 \mathrm{~nm}$ and high power, one can induce permanent erasure of the modulated grating component and that this approach could be used to anneal a Type $1 \mathrm{~A}$ grating.

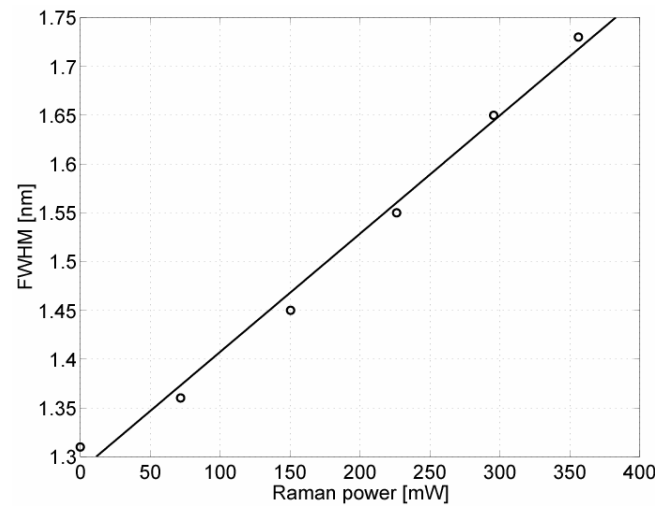

Fig. 9. Linear relation between the FWHM bandwidth of a Type IA grating and the intensity of the Raman laser.

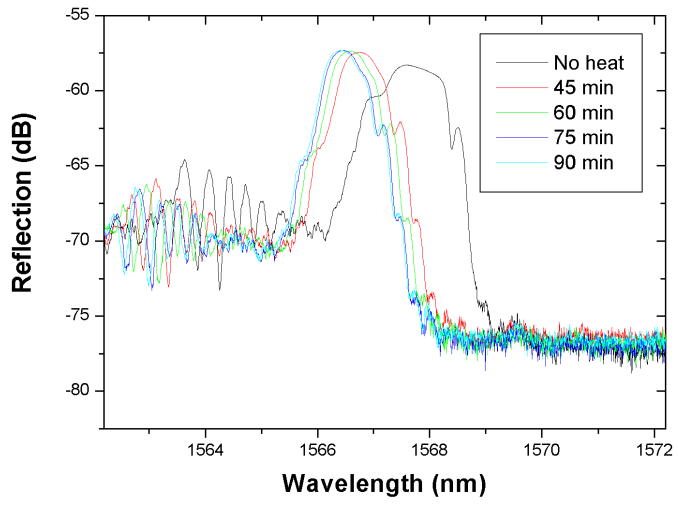

Fig. 10. Permanent and partial laser induced erasure of the Type 1A grating with time.

\section{CONCLUSIONS}

We have shown that it is possible to selectively heat a grating within an array of other gratings by purely optical methods, inducing reversible wavelength shifts, chirp and controlled permanent grating decay (annealing). Since all grating types can be written in a section of pre-exposed fibre this method of optical tuning could be used for all existing Bragg grating applications making the technique invaluable to a multitude of applications such as selective wavelength tuning in a DFB laser or edge filter modification in a sensing application. It is possible to tailor the absorption of the preexposed section to mirror the decay in intensity resulting in a uniform heating of the grating. However, this would alter the mean fibre index along the pre-exposed section, inducing a potentially large (up to 20nm) chirp across the grating.

\section{ACKNOWLEDGEMENTS}

The authors acknowledge the UK DTI-EPSRC LINK project EMPIRE. KK and AGS respectively acknowledge the Higher Technical Institute - Promotion of Research Fund and studentship of the UK EPSRC and BAE Systems.

\section{REFERENCES}

1. Y. Liu, J.A.R. Williams, L. Zhang and I. Bennion, "Abnormal spectral evolution of fibre Bragg gratings in hydrogenated fibres," Optics Letters Vol. 27, p 586-588, 2002.

2. A.G. Simpson, K. Kalli, K. Zhou, L. Zhang and I. Bennion, "Formation of type IA fibre Bragg gratings in germanosilicate optical fibre," Electronics Letters, Vol. 40, p 163-164, 2004.

3. A.G. Simpson, K. Kalli, L. Zhang, K. Zhou and I. Bennion, "Abnormal photosensitivity effects and the formation of type IA FBGs," BGPP, Monterey, California, MD31, 2003.

4. A.G. Simpson, K. Kalli, K. Zhou, L. Zhang and I. Bennion, "An idealised method for the fabrication of temperature invariant IA-I strain sensors," postdeadline session, OFS16 Nara, Japan, PD4, 2003.

5. P.J. Lemaire, R.M. Atkins, V. Mizrahi and W.A. Reed, "High-pressure H-2 loading as a technique for achieving ultrahigh UV photosensitivity and thermal sensitivity in GeO2 doped optical fibres,” Electronics Letters, Vol. 29, p 1191-1193, 1993. 